Revue internationale de pédagogie de

l'enseignement supérieur

28(1) | 2012

Numéro spécial - printemps 2012

\title{
La professionnalisation de l'offre de formation universitaire : quelques spécificités
}

Richard Wittorski

\section{(2) OpenEdition}

Édition électronique

URL : http://journals.openedition.org/ripes/580

ISSN : 2076-8427

Éditeur

Association internationale de pédagogie universitaire

Référence électronique

Richard Wittorski, «La professionnalisation de l'offre de formation universitaire : quelques

spécificités », Revue internationale de pédagogie de l'enseignement supérieur [En ligne], 28(1) | 2012, mis en ligne le 20 avril 2012, consulté le 07 septembre 2020. URL : http://journals.openedition.org/ripes/ 580

Ce document a été généré automatiquement le 7 septembre 2020

Article L.111-1 du Code de la propriété intellectuelle. 


\title{
La professionnalisation de l'offre de formation universitaire : quelques spécificités
}

\author{
Richard Wittorski
}

\section{Introduction}

1 Comme nous l'évoquions ailleurs (Wittorski, 2008), la vocation professionnelle de la formation universitaire n'est pas nouvelle. Ainsi, depuis ses origines, la formation des médecins, par exemple, relève d'une logique d'articulation forte avec les situations professionnelles. Il en va de même pour les formations universitaires de type diplôme universitaire de technologie (DUT) délivré par les instituts universitaires de technologie (IUT) en France qui ont cette vocation affirmée, depuis leur création, même si elles ont eu longtemps un statut de filières à part dans les universités se réclamant, dans l'ensemble, d'une logique de production et de diffusion des savoirs scientifiques et techniques. Nous assistons, au contraire, aujourd'hui à un mouvement de généralisation de la professionnalisation (et c'est ici que se situe la nouveauté) dans lequel la norme devient précisément la visée professionnalisante de l'offre de formation (à l'image de la mission confiée par les derniers textes officiels à l'université française). Dans ce contexte, il est donc utile de prendre le temps d'analyser quelquesunes des spécificités de cette professionnalisation dans un cadre universitaire en s'appuyant sur l'étude de plusieurs pratiques disponibles.

2 Pour ce faire, notre propos sera organisé en trois temps : tout d'abord, nous essaierons de mieux circonscrire le sens de l'objet "professionnalisation", ensuite, nous étudierons quelques caractéristiques fortes des dispositifs proposés aujourd'hui dans un cadre universitaire ou dans les formations dites supérieures, enfin nous évoquerons les conditions qui nous semblent devoir être réunies pour mettre en œuvre une formation professionnelle et/ou professionnalisante (cette dernière partie nous conduira à changer de posture, de celle de chercheur à celle d'acteur social). 


\section{Qu'entend-on par professionnalisation?}

3 Comme le note Paradeise (2003), les mots profession et professionnalisation apparaissent dans un contexte de marché libre où les acteurs économiques ressentent le besoin de développer une rhétorique concernant leur contribution au marché pour conquérir et accroître leur place. C'est probablement à ce niveau qu'il convient de situer l'apparition, dès le début du $20^{\mathrm{e}}$ siècle, du mot profession dans les pays anglosaxons, associé d'ailleurs à l'image de la profession libérale. En France, il apparaît dans un contexte différent qui est caractérisé par un État hiérarchique. (En France, traditionnellement, l'État est très présent dans l'organisation d'un certain nombre d'activités et donc d'emplois qui font l'objet d'un classement hiérarchique). Dès lors, la profession ne repose pas tant sur le modèle de la profession libérale mais davantage sur celui des corps d'État.L'enjeu est donc, ici, l'organisation collective et la mise en reconnaissance de soi dans l'environnement à des fins de conquête d'une meilleure place dans une hiérarchie étatique.

4 Les enjeux portés par les organisations (entreprises) ne concernent pas, selon nous, la constitution des professions mais la professionnalisation des salariés entendue comme une intention d'accompagner la flexibilité du travail (modification continue des compétences en lien avec l'évolution des situations de travail). Ainsi, les caractéristiques des nouvelles organisations (production tirée par l'aval, intégration des activités et décloisonnement des fonctions) qui apparaissent dans ces 40 dernières années génèrent trois conséquences: 1) l'élargissement et l'enrichissement des compétences et des tâches; 2) la réduction de la ligne hiérarchique; et 3) le développement du caractère collectif du travail.

5 Au total, le mot professionnalisation demeure fortement polysémique ;son usage est large et les sens qui lui sont attribués sont multiples. Nous identifions ainsi au moins trois sens à l'œuvre dans les pratiques sociales :

1. La professionnalisation-profession: sens utilisé par les acteurs sociaux où il s'agit de se constituer comme profession ;

2. La professionnalisation-efficacité du travail : sens utilisé par les organisations où il s'agit de développer la flexibilité des personnes au travail et, au final, leur plus grande efficacité ;

3. La professionnalisation-formation : sens utilisé par les milieux de la formation où il s'agit de favoriser le développement des savoirs et des compétences.

6 En lien avec ce troisième sens, s'agissant de l'enseignement supérieur français, le mouvement de professionnalisation de la formation a été récemment réactivé et significativement étendu, par exemple, à la faveur de la mise en place des licences professionnelles (Maillard \& Veneau, 2003), instaurées en 1999, pour répondre aux besoins spécifiques de bassins ou d'entreprises locales. Au plan européen, la déclaration de Bologne, énoncée en 1999 et signée par vingt neuf pays européens, promeut, d'une part, un schéma d'organisation des études supérieures (en France, le système LMD pour «Licence, Master et Doctorat ») et, d'autre part, un schéma de validation des contenus permettant de capitaliser des crédits dans des établissements différents.

7 Par ailleurs, le Conseil européen de Lisbonne, en 2000, constitue une autre étape significative, notamment à partir du projet de coopération renforcée pour l'éducation et la formation professionnelle intitulé «Processus de Bruges-Copenhague ». Celui-ci 
institue l'European Credit in Vocational Education and Training (ECVET), un système européen d'accumulation et de transfert d'unités capitalisables pour l'enseignement professionnel qui suppose la mise en place de normes définies en termes de compétences ainsi que l'European Qualifications Framework (EQF), un cadre européen de qualification destiné à permettre le positionnement européen des certifications nationales et à articuler les cadres nationaux de certification présents ou à venir.

Cette incitation et valorisation politique d'une professionnalisation accrue des formations s'est traduite, dans les pratiques, à la fois par une diversification des spécialités enseignées comme le notent Béduwé, Espinasse et Vincens (2007) et par une tendance à la conception de curricula à base d'approche par compétence (l'exemple significatif à cet égard étant celui du Québec : voir les travaux de Monchatre, 2007).

\section{Quelques leviers utilisés par les formations universitaires professionnalisantes ${ }^{1}$}

Analysons quelques exemples de formations supérieures professionnalisantes - la formation supérieure professionnelle des enseignants et des travailleurs sociaux, l'introduction de la validation des acquis d'expérience (VAE) et ses conséquences - et précisons, à chaque fois, les leviers utilisés pour professionnaliser.

\subsection{La formation professionnelle des enseignants français en Institut universitaire de formation des maîtres (IUFM)}

10 La formation proposée jusqu'en 2010, avant la réforme, dite de la "masterisation ${ }^{2}$ » de la formation des enseignants, en IUFM aux enseignants stagiaires français reçus aux concours du second degré (Agrégation ou Certificat d'aptitude au professorat de l'enseignement secondaire (Capes)) repose sur le principe de l'alternance: les stagiaires, placés dans des collèges ou lycées, prennent en charge une ou plusieurs classes sur une année complète pendant laquelle ils sont, par ailleurs, présents à l'IUFM chaque semaine pour une à deux journées de formation. Par différence avec d'autres pays, tel le Québec par exemple, la formation des enseignants français, avant 2010, est donc confiée à des instituts spécifiques et non aux départements d'éducation des universités.

11 La formation délivrée à l'IUFM est d'abord dite de nature disciplinaire (savoirs relatifs aux disciplines enseignées), mais également dite générale (savoirs dits transversaux ou d'ordre pédagogique). Elle utilise enfin, à des fins de professionnalisation, le levier de l'analyse des pratiques.

12 À partir de quelques travaux empiriques réalisés, il semble que l'analyse de pratiques constitue un outil privilégié de professionnalisation dans la mesure où elle assureune articulation étroite entre le travail et la formation (l'acte de travail devient objet et occasion de formation, dès lors qu'il fait l'objet d'une analyse par son auteur). Elle permet à la fois un retour sur l'action et la transformation des actions habituellement mobilisées au travail en connaissances sur l'action, traduisant ainsi un apprentissage à partir de l'action qui participe de la construction de l'expérience des sujets. Nous avons particulièrement mis en évidence ces dynamiques dans une recherche concernant les modalités et les effets des dispositifs d'analyse des pratiques proposés aux enseignants 
(Wittorski, 2003). Il apparait ainsi que dans l'activité professionnelle, les enseignants stagiaires développent des façons d'agir sur le tas, dans l'immédiateté, dans l'instant de la situation (qui ont souvent un statut d'imprévu). Bien souvent d'ailleurs, ces pratiques sont des formes d'ajustement particulier des choix effectués en amont qui s'avèrent, dans l'instant de leur mise en œuvre, inadéquats et nécessitant une adaptation (le public n'étant pas, par exemple, dans les dispositions souhaitées ou prévues, ...). Ces «trucs de métier» ont pour particularité d'être produits dans la situation et donc d'être efficaces dans l'instant, mais rarement reproductibles à l'identique dans d'autres situations, car ils sont adaptés à des contextes particuliers. Ce que permet précisément l'analyse de pratiques, c'est d'offrir un espace de mise en mots de ces pratiques spontanées qui, sans ce lieu de parole, resteraient souvent incorporées à l'action, c'està-dire non identifiées par leurs auteurs (voir la notion de compétence incorporée ${ }^{3}$ développée par Leplat (1995)). L'analyse de pratiques repose souvent sur une démarche de groupe consistant à réunir des personnes partageant les mêmes activités et qui sont incitées à décrire, tour à tour, des pratiques professionnelles vécues (vis-à-vis desquelles ils ressentent souvent une difficulté), celles-ci sont ensuite discutées et analysées en groupe. L'analyse de pratiques permet alors d'identifier les pratiques incorporées à l'action, mais aussi, par le travail d'échange collectif, d'en saisir les tendances communes, les principes qui les organisent, ces derniers étant utiles pour développer d'autres pratiques de retour en situation professionnelle. C'est ce que nous entendons par principe organisateur des pratiques, des règles d'action (à validité individuelle ou collective) régissant les pratiques professionnelles et susceptibles d'en générer d'autres. Prenons quelques exemples extraits des discours des enseignants stagiaires qui nous semblent renvoyer à ces principes organisateurs des pratiques. Ces extraits sont tirés de la recherche mentionnée ci-dessus (Wittorski, 2003) :

- «Il faut gérer ses émotions en classe». Ce principe est identifié par certains stagiaires après avoir fait un travail d'analyse de leurs propres difficultés de gestion de leurs émotions en classe lors du dispositif d'analyse de pratiques ;

- « Il faut se mettre en scène ». Ce principe est exprimé par certains stagiaires après avoir analysé des moments d'interaction avec la classe qui avaient posé problème.

\subsection{Des formations professionnelles continues de travailleurs sociaux}

13 Les dispositifs français de Diplôme des hautes études et pratiques sociales (DHEPS) et Diplôme supérieur de travail social (DSTS) (appelé aujourd'hui Diplôme d'Etat d'ingénierie sociale (DEIS)), comparables, par exemple, à la maîtrise de service social proposée au Québec par l'Université de Sherbrooke,s'adressent en dominante à des publics de travailleurs sociaux salariés, donc déjà engagés dans une activité professionnelle. Ces dispositifs utilisent explicitement, de pair avec des activités de formation plus classiques, le levier de la recherche-action professionnelle qui est sollicitée à l'occasion de la réalisation du mémoire (DSTS et DHEPS). Le travail demandé consiste souvent à définir une question-problème rencontrée par le stagiaire dans son exercice professionnel (en lien avec sa pratique), à mener ensuite un travail théorique et empirique à son sujet en vue de mieux comprendre ses contours et à proposer, enfin, des réponses "éclairées" susceptibles d'être transférées dans l'espace professionnel (plus tard, s'il s'agit d'un mémoire). 

professionnelle peut constituer un outil utile pour professionnaliser ou reprofessionnaliser des acteurs placés dans un parcours de mobilité professionnelle. Cela correspond à un enjeu réel, notamment pour les catégories professionnelles dont les contextes d'activités bougent et qui doivent rompre avec leurs pratiques habituelles pour produire de nouvelles façons de travailler. Ainsi, les travailleurs sociaux, les animateurs et les agents de formation sont de plus en plus sollicités pour apporter des réponses nouvelles et spécifiques à des situations inédites. Il leur faut alors produire de nouvelles stratégies d'action. Cette capacité à la production de changement peut être développée si les acteurs ont élaboré de nouvelles capacités à la prise de recul, à l'analyse ... De ce point de vue, l'engagement dans une démarche de formation appelée «recherche-action » ou "recherche-action professionnelle » ancrée sur les pratiques professionnelles, est, probablement, un moyen permettant de produire ces nouvelles capacités.

De façon provisoire, nous dirons que la recherche-action professionnelle s'apparente à une démarche constructiviste: l'action, en se faisant, génère de nouvelles façons de faire et l'analyse de cette action conduit l'individu à produire un cadre de référence analytique de l'action (et non seulement un cadre de référence pour l'action) qui laisse des traces sur la poursuite de l'engagement dans la situation. Il s'agit donc d'un processus itératif doublement constructiviste dans la mesure où il transforme conjointement l'action et son agent par la production d'outils mentaux nouveaux d'accompagnement et de gestion du changement. point commun de l'acte professionnel, de l'acte de recherche et de la recherche-action professionnelle est la constitution d'un cadre de représentation de l'action. Celui-ci n'a pas pour autant les mêmes fonctions dans les trois cas : il permet d'organiser/diriger l'action au moment où elle se déroule (acte professionnel); il permet de lire et de comprendre l'action observée (acte de recherche); et il permet conjointement d'analyser, de questionner et d'organiser l'action en cours (recherche-action professionnelle). 
Tableau 1 : Acte de recherche, acte professionnel et recherche action professionnelle

\begin{tabular}{|c|c|c|c|}
\hline & Acte de recherche & Acte professionnel & $\begin{array}{c}\text { Acte de recherche-action } \\
\text { professionnelle }\end{array}$ \\
\hline \multirow{2}{*}{$\begin{array}{l}\text { Construction d'un } \\
\text { cadre pour lire, agir } \\
\text { sur ou penser l'action }\end{array}$} & $\begin{array}{l}\text { Construction d'un cadre } \\
\text { théorique préalable ou en } \\
\text { cours de recueil } \\
\text { d'informations }\end{array}$ & $\begin{array}{l}\text { Production d'un cadre } \\
\text { fonctionnel } \\
\text { (représentations } \\
\text { fonctionnelles) } \\
\text { accompagnant l'action }\end{array}$ & $\begin{array}{l}\text { Conceptualisation de } \\
\text { l'action en cours de } \\
\text { réalisation }\end{array}$ \\
\hline & $\begin{array}{c}\text { Il a une fonction de } \\
\text { lecture/analyse de l'action }\end{array}$ & $\begin{array}{c}\text { Il a une fonction } \\
\text { d'organisation de l'action }\end{array}$ & $\begin{array}{l}\text { Fonction double de } \\
\text { questionnement/analyse et } \\
\text { d'organisation de l'action }\end{array}$ \\
\hline \multirow{2}{*}{$\begin{array}{c}\text { Statut et position de } \\
\text { l'acteur par rapport à } \\
\text { l'action }\end{array}$} & Action observée & $\begin{array}{l}\text { Action en cours de } \\
\text { réalisation }\end{array}$ & $\begin{array}{l}\text { Engagement " réfléchi } » \\
\text { (" réflexif } ») \text { dans l'action }\end{array}$ \\
\hline & $\begin{array}{l}\text { Le chercheur est extérieur à } \\
\text { l'action }\end{array}$ & $\begin{array}{c}\text { Le professionnel est auteur } \\
\text { de l'action }\end{array}$ & $\begin{array}{l}\text { Le chercheur et le } \\
\text { professionnel s'impliquent } \\
\text { et prennent du recul par } \\
\text { rapport à l'action }\end{array}$ \\
\hline
\end{tabular}

\subsection{L'introduction de la VAE dans les universités françaises}

17 L'introduction de la validation des acquis d'expérience (VAE) dans les universités françaises a contribué plus ou moins significativement, non sans réserves et résistances dans un certain nombre de cas, à un mouvement de professionnalisation de l'offre de formation. Elle a suscité d'abord la réécriture des objectifs de formation en compétences de manière à penser des équivalences avec les expériences professionnelles des candidats à la VAE. Cette réécriture a parfois été également l'occasion de réorienter de façon significative les contenus ou modalités de formation choisis en lien avec les objectifs de compétences - et non plus seulement de savoirs visés; dans d'autres cas, les contenus et modalités de formation sont demeurés inchangés.

Prenons l'exemple du Diplôme universitaire de formateur conseil (Dufco) de l'Université Paris Descartes. Ce diplôme pourrait être comparé, par exemple, au diplôme de $2^{\text {ème }}$ cycle en gestion de l'éducation et de la formation proposé par l'Université de Sherbrooke au Québec: comme le Dufco, il vise à professionnaliser les pratiques et couvre l'ensemble du processus de gestion de la formation, de l'analyse des besoins à l'évaluation des impacts.

19 S'agissant du Dufco, la nécessité de penser une articulation avec la licence professionnelle et le master existants, mais aussi de prendre en compte des demandes de VAE croissantes, ont conduit à réécrire, en 2004, les objectifs de formation en termes de compétences et à proposer une architecture de formation intégrant une nouvelle modalité consistant en la conduite d'un projet tutoré relevant du conseil en formation nécessitant l'adoption, par les stagiaires, d'une posture de consultant s'appuyant sur 
les apports de la formation. Autrement dit, et de façon plus large probablement, la VAE nous semble être une occasion qui peut concourir à la professionnalisation plus forte de l'offre de formation.

Par ailleurs, et sur un autre plan, celui de la professionnalisation des personnes, même si la VAE ne constitue pas à proprement parler un dispositif de formation formelle professionnalisant, le parcours de constitution du dossier semble produire sur le candidat des effets formateurs non négligeables qui induisent une réappropriation de son expérience et une reconstruction de son identité professionnelle. Le moyen utilisé ici est celui de l'écriture sur la pratique qui caractérise en dominante le processus de constitution du dossier de candidature à la validation (Magnier \& Werthe, 2001).

\subsection{L'alternance conjuguée au retour réflexif}

21 Ces exemples montrent que les leviers qui sont particulièrement mobilisés pour accompagner ou susciter la professionnalisation des individus dans les formations supérieures relèvent :

- D'une part, de l'alternance : de ce point de vue, pas de formation universitaire dite professionnalisante sans un temps plus ou moins long d'exercice professionnel en alternance avec un parcours de formation dans lequel l'action professionnelle nouvelle en cours de construction est, elle-même, régulièrement réinterrogée ;

- D'autre part, d'une analyse orale ou écrite des pratiques professionnelles (il s'agit tantôt de groupes d'analyse de pratiques, tantôt de travaux d'écriture sur la pratique (mémoire professionnel, recherche-action...).

Ces deux leviers sont, bien entendu, souvent fortement articulés dans les offres de formation. À côté des savoirs théoriques, ils permettent de "travailler » des savoirs non théoriques que l'on appelle souvent des savoirs d'action (Barbier \& Galatanu, 2004) ou encore des savoirs professionnels. Par exemple, le fait de réinterroger en formation l'action professionnelle mise en œuvre in situ permet de changer le statut de l'action pour son auteur: ainsi devient-elle pour lui un acquis «su» de l'expérience, alors même que l'action considérée pouvait être fortement incorporée à la situation (et donc non identifiée par le sujet comme nous le disions plus haut).

Les leviers, ici mentionnés, sont fréquemment utilisés dans les formations universitaires mais souvent ils ont tendance à s'accompagner d'une conception demeurant en grande partie applicative ou déductive des rapports travail formation consistant à séparer travail et formation et à consacrer la suprématie de la théorie sur la pratique : la logique de l'offre de formation formelle prédomine. Par exemple, ce qui fait l'objet d'échange en séance d'analyse de pratiques est rarement utilisé pour réinterroger les contenus et modalités de formation, considérant que l'analyse de pratiques intéresse uniquement les apprentissages relevant de la sphère professionnelle. De façon liée, on constate également souvent au niveau master en France, par exemple, des schémas d'alternance prévoyant le temps de stage uniquement en fin de formation. 


\section{La professionnalisation comme combinaison de voies}

24 Comment dépasser ces difficultés? Comment professionnaliser davantage l'offre de formation universitaire? Changeons maintenant de posture pour réfléchir à ces deux questions ; de chercheur-analyste de pratiques sociales, adoptons celle d'acteur social et demandons-nous à quelles conditions l'offre de formation universitaire peut-elle devenir plus professionnalisante ou, du moins, mettre en œuvre une conception moins "applicative» des rapports travail-formation. Nous commencerons par préciser la différence que nous proposons d'introduire, dans le prolongement des travaux de Sonntag (2007), entre formation professionnelle et formation professionnalisante; ensuite, nous développerons l'idée selon laquelle une formation professionnelle ou professionnalisante devrait s'appuyer explicitement sur une combinaison de voies de professionnalisation, c'est-à-dire une combinaison de formats de situations apprenantes.

\subsection{Formation professionnelle et formation professionnalisante}

Il nous semble tout d'abord essentiel de faire une différence claire, à la manière de Sonntag (2007), entre ce qui relève d'une logique de formation professionnelle et ce qui relève d'une logique de formation professionnalisante, tenant compte du fait que le statut d'un certain nombre de savoirs en jeu n'est alors pas de même nature dans l'un et l'autre cas.

Une formation professionnelle forme son public aux outils, méthodes, techniques utiles pour agir. L'offre de formation s'emploie alors à proposer des situations apprenantes susceptibles de faciliter le développement d'apprentissages méthodologiques et techniques permettant aux stagiaires de détenir progressivement les savoirs mais également les méthodes de travail permettant de traiter efficacement les situations professionnelles qui leur seront proposées dans l'exercice ordinaire de leur activité à venir. Nous pourrions dire que les apprentissages visés sont bien entendu de l'ordre de savoirs théoriques et scientifiques mais également de savoirs d'action ou pratiques au sens où il s'agit d'énoncés concernant des façons de faire jugées efficaces (le critère de validation est bien ici celui de l'efficacité de l'action (Wittorski, 2007)).

Par différence, nous dirons qu'une formation professionnalisante, certes forme également aux outils, techniques nécessaires pour agir dans l'exercice ordinaire du métier, mais cette transmission se réalise en référence aux conduites professionnelles jugées légitimes par le groupe professionnel. Une formation professionnalisante a donc pour enjeu la construction identitaire, le rapport au métier en lien avec l'identité collective. Elle nécessite la constitution préalable ou simultanée d'un groupe professionnel capable de tenir un discours sur les bonnes pratiques. Or, il faut bien le dire s'agissant de nombreuses activités professionnelles relevant notamment du champ des services, les institutions employeurs jouent souvent un rôle plus important dans la définition des repères professionnels que les groupes professionnels eux-mêmes qui ont souvent du mal à exister et à faire entendre une voix homogène et jugée légitime aux yeux des membres de la profession et des institutions employeurs (exemple des enseignants, des travailleurs sociaux). Dans ce cas, la formation proposée relève 
souvent davantage d'une formation professionnelle qui ne fait pas d'ailleurs l'objet d'une reconnaissance évidente et univoque par les milieux professionnels.

Par différence avec les formations professionnelles, nous pourrions dire que les apprentissages visés dans une formation professionnalisante sont bien entendu de l'ordre de savoirs théoriques et scientifiques mais également de savoirs professionnels (Wittorski, 2007) plutôt que de savoirs d'action, au sens où il s'agit, là, d'énoncés concernant des façons de faire jugées non plus seulement efficaces mais également légitimes(le critère de validation est bien ici celui de la légitimité de l'action au regard des us et coutumes du groupe professionnel et de l'optimisation de sa reconnaissance sociale).

Tableau 2 : Formation professionnelle et formation professionnalisante

\begin{tabular}{|c|c|}
\hline Formation professionnelle & Formation professionnalisante \\
\hline Savoirs d'action & Savoirs professionnels \\
\hline Critère d'efficacité & Critère de légitimité \\
\hline
\end{tabular}

On le pressent, une formation professionnalisante s'ancre sur un genre professionnel à la façon de Clot (2007), qui donne une légitimité forte aux contenus de formation délivrés dès lors que les représentants du milieu professionnel concerné sont, par ailleurs, impliqués dans l'accompagnement (tutorat) et l'évaluation des apprentissages. Qu'il s'agisse d'une formation professionnelle ou d'une formation professionnalisante, les dispositifs proposés doivent réfléchir aux formats de situations qu'ils proposent en fonction des apprentissages qu'ils souhaitent développer.

\subsection{Penser une combinaison de voies}

Nos propres travaux engagés depuis une vingtaine d'années dans le champ des rapports travail-formation, nous ont conduit à formaliser, au fur et à mesure des terrains de recherche investigués, une grille de lecture des voies de la professionnalisation qui a déjà largement été présentée (notamment Wittorski, 1997, 2003, 2007).

Nous entendons par voies de la professionnalisation des formats spécifiques de situations apprenantes observées dans des situations de travail et/ou de formation proposées aux individus (formation par alternance, mise au travail) qui conduisent à développer des apprentissages particuliers et distincts d'une voie à l'autre. Nous avons ainsi identifié six voies de professionnalisation rappelées succinctement ici :

1. La logique de l'action (formation sur le tas) qui caractérise le fait que des sujets sont en prise avec une situation familière mais présentant un caractère de nouveauté (utilisation de nouveaux outils, par exemple). Cela les conduit alors à modifier leurs façons habituelles de faire le plus souvent sans qu'ils ne s'en rendent compte par un ajustement au fil de l'action (compétence incorporée, Leplat, 1995) ;

2. La logique de la réflexion et de l'action (formation alternée)caractérise les situations dans lesquelles les individus sont face à des problèmes inédits qui les conduisent à construire, pas à pas, un procès d'action intellectualisé ou mentalisé au sens où il fait l'objet d'un accompagnement réflexif ; 
3. La logique de la réflexion sur l'action (analyse de pratiques)correspond aux moments où les individus analysent de façon rétrospective leur action: ils mettent en mots un certain nombre de principes participant ainsi à l'enrichissement de leur patrimoine d'expérience. Ils développent, à cette occasion, une compétence tournée vers l'analyse de leur action et des connaissances et/ou savoirs sur l'action ;

4. La logique de la réflexion pour l'action (analyse de pratiques également) caractérise des moments individuels ou collectifs de définition, par anticipation, de nouvelles façons de faire dans l'intention (fréquemment observée) d'être plus efficace au travail. Cette voie permet de développer des savoirs pour l'action (préparatoires d'actions nouvelles) ;

5. La logique de la traduction culturelle par rapport à l'action (accompagnement) correspond, par exemple, aux situations de tutorat: le tuteur transmet des savoirs/connaissances mais aussi aide le tutoré à prendre du recul, à modifier ses façons habituelles de voir et de penser l'action et la situation (notion de traduction ${ }^{4}$ culturelle). Il contribue ainsi, sur le plan identitaire, à la fois à transmettre des éléments de la culture professionnelle et à orienter le positionnement professionnel, tout du moins la façon dont l'individu conçoit les situations et ses propres pratiques ainsi que celles des autres;

6. La logique de l'intégration assimilation (formation magistrale) caractérise des situations dans lesquelles les individus utilisent des ressources documentaires ou visuelles pour acquérir des savoirs ou des connaissances plutôt de type théorique. probablement plus largement le cas des apprentissages portant sur la relation humaine et institutionnelle (jeux de pouvoir, positionnement professionnel). Ainsi, tout l'enjeu d'une formation professionnelle ou professionnalisante serait d'organiser, en fonction des apprentissages visés, une combinaison de voies pertinente qui, on le voit, ne peut plus reposer sur une conception applicative ou déductive des rapports travailformation mais plutôt sur une conception itérative (voir plus loin). Cette combinaison donnera d'ailleurs une place plus ou moins grande au terrain, via par exemple le tutorat et les modalités de l'évaluation, pour légitimer les apprentissages visés selon que l'on a affaire à une formation professionnelle ou à une formation professionnalisante. 


\section{Conclusion}

Une formation universitaire professionnelle et/ou professionnalisante suppose donc probablement d'orchestrer une combinaison singulière de voies selon les apprentissages visés. Bien entendu, est ici alors posée la question de la temporalité des modalités pédagogiques : tel moment de formation intervient-il trop tôt ou trop tard ou au bon moment par rapport au vécu professionnel. Une formation universitaire ayant pour intention de professionnaliser doit, en outre, selon nous, s'appuyer sur une analyse préalable du travail réel pour identifier les compétences à développer en formation dont la nécessité d'identifier les compétences visées et les compétences validées par le secteur professionnel. Elle suppose également de penser la formation par finalité d'action. Or, on pense souvent spontanément la formation par apports disciplinaires. Elle suppose ensuite une diversification des modalités pédagogiques : au delà du cours -les compétences ne s'enseignent pas, loin de constituer des énoncés, elles sont souvent considérées comme étant des modalités d'action en situation-, prévoir des mises en situation, propices au développement des compétences, et des moments d'analyse de pratiques, pour leur exploitation. Elle nécessite enfin de mettre en place une évaluation des compétences, c'est-à-dire une évaluation des modalités d'action effectives des sujets et non seulement une évaluation des savoirs, c'est-à-dire des énoncés déclaratifs sur des propriétés d'objets ou d'actions. Cela conduit à partager le pouvoir de conception de la formation avec les milieux professionnels tout en faisant évoluer le métier de formateur, un formateur capable d'élaborer des situations problèmes, de développer l'analyse de pratiques.

Bien plus, une formation professionnalisante nous semble donc devoir s'appuyer sur une conception intégrative ou itérative de l'alternance et des rapports travailformation en lien avec un paradigme différent susceptible de penser la continuité et la complémentarité entre les deux espaces du travail et de la formation. Les individus sont alors invités à développer une posture réflexive rétrospective par rapport à leur action permettant de transformer une expérience en actes en une expérience sue. L'analyse de pratiques articule alors les deux espaces du travail et de la formation. On parle non seulement de savoirs théoriques mais aussi de savoirs d'action.

\section{BIBLIOGRAPHIE}

Barbier, J.-M., \& Galatanu, O. (2004). Les savoirs d'action : une mise en mot des compétences? Paris : L'Harmattan.

Béduwé, C., Espinasse, J.-M., \& Vincens, J. (2007). De la formation professionnelle à la professionnalité d'une formation. Formation Emploi, 99, 103-121.

Clot, Y. (2007). De l'analyse des pratiques au développement des métiers, Education et didactique, 1, 83-93.

Leplat, J. (1995). À propos des compétences incorporées. Éducation Permanente, 123, 101-114. 
Magnier, J., \& Werthe, Ch. (2001). L'expérience revisitée à l'occasion de la VAP. Formation Emploi, 75, 29-41.

Maillard, D., \& Veneau, P. (2003). Les licences professionnelles. Bref-Céreq, 197, 1-4.

Monchatre, S. (2007). En quoi la compétence devient-elle une technologie sociale ? Réflexions à partir de l'expérience québécoise. Formation Emploi, 99, 29-45.

Paradeise, C. (2003). Comprendre les professions : l'apport de la sociologie. Sciences Humaines, 139, 26-29.

Sonntag, M. (2007). Les formations d'ingénieurs : des formations professionnelles et professionnalisantes. Orientations, contenus, contextes. Recherche et Formation, 55, 11-26.

Wittorski, R. (1997). Analyse du travail et production de compétences collectives. Paris : L'Harmattan. Wittorski, R. (2003). Analyse de pratiques et professionnalisation des enseignants. CNAM : Centre de Recherche sur la Formation.

Wittorski, R. (2007). Professionnalisation et développement professionnel. Paris : L'Harmattan.

Wittorski, R. (2008). Professionnalisation et offre de formation en enseignement supérieur. Dans P. Hébrard \& C. Solar (dir.), Professionnalisation et formation des adultes : une perspective universitaire France-Québec (p. 47-70). Paris : L'Harmattan.

\section{NOTES}

1. Cette partie de l'article est très largement inspirée de Wittorski (2008).

2. Cette réforme vise maintenant à recruter les enseignants au niveau master (baccalauréat Français +5 ans d'études supérieures) alors que le recrutement se faisait, avant 2010, à partir de la licence française (baccalauréat Français +3 ans d'études supérieures), quelle que soit la discipline.

3. J. Leplat définit une compétence incorporée comme "faisant corps avec les actions qui les expriment, [...] elles sont facilement accessibles, difficilement verbalisables, peu coûteuses sur le plan de la charge mentale, difficilement dissociables, très liées au contexte» (Leplat, 1995, p. 102).

4. Notion de «traduction » en référence aux travaux de la Sociologie de la Traduction de Bruno Latour et Michel Callon.

\section{RÉSUMÉS}

Quelle est l'offre de formation qui, aujourd'hui, ne se réclame pas d'une visée professionnalisante? En France, à la fois portée par les orientations nationales et européennes s'agissant de l'organisation de la formation initiale et continue et prônée par les secteurs privés, la professionnalisation fait d'évidence l'objet à la fois d'une attente sociale et d'une valorisation fortes.

Cette contribution a pour intention à la fois de mentionner quelques spécificités de la professionnalisation de l'offre de formation universitaire et de préciser ce qui nous semble 
constituer les conditions d'une professionnalisation plus forte, illustrée dans nos propos par la formation des enseignants et la formation continue des travailleurs sociaux.

A travers ces spécificités, Il s'agira, ainsi, de mieux cerner la nature des difficultés à «faire vivre » l'intention de professionnalisation, et, enfin, dans ce contexte encourager la combinaison de « six voies de professionnalisation » (Wittorski, 2007).

\section{INDEX}

Mots-clés : formation professionnalisante, formation professionnelle, formation universitaire professionnalisante, professionnalisation, voies de professionnalisation

\section{AUTEUR}

\section{RICHARD WITTORSKI}

Professeur des universités,

Conservatoire national des arts et métiers (CNAM), école management et société

2 rue conté 75003 Paris

richard.wittorski@cnam.fr 